\title{
Detection and Elimination of a Potential Fire in Engine and Battery Compartments of Hybrid Electric Vehicles
}

\author{
Macam S. Dattathreya, ${ }^{1}$ Harpreet Singh, ${ }^{2}$ and Thomas Meitzler ${ }^{1}$ \\ ${ }^{1}$ Tank Automotive Research, Development and Engineering Center, Warren, MI 48397, USA \\ ${ }^{2}$ Department of Electrical and Computer Engineering, Wayne State University, Detroit, MI 48202, USA
}

Correspondence should be addressed to Macam S. Dattathreya, msdattathreya@yahoo.com

Received 3 May 2012; Accepted 13 October 2012

Academic Editor: Ashu M. G. Solo

Copyright ( 2012 Macam S. Dattathreya et al. This is an open access article distributed under the Creative Commons Attribution License, which permits unrestricted use, distribution, and reproduction in any medium, provided the original work is properly cited.

\begin{abstract}
This paper presents a novel fuzzy deterministic noncontroller type (FDNCT) system and an FDNCT inference algorithm (FIA). The FDNCT uses fuzzy inputs and produces a deterministic non-fuzzy output. The FDNCT is an extension and alternative for the existing fuzzy singleton inference algorithm. The research described in this paper applies FDNCT to build an architecture for an intelligent system to detect and to eliminate potential fires in the engine and battery compartments of a hybrid electric vehicle. The fuzzy inputs consist of sensor data from the engine and battery compartments, namely, temperature, moisture, and voltage and current of the battery. The system synthesizes the data and detects potential fires, takes actions for eliminating the hazard, and notifies the passengers about the potential fire using an audible alarm. This paper also presents the computer simulation results of the comparison between the FIA and singleton inference algorithms for detecting potential fires and determining the actions for eliminating them.
\end{abstract}

\section{Introduction}

A hybrid electric vehicle (HEV) propulsion system uses a high-voltage battery and an engine. The engine is located in front of the vehicle, and the battery is in the back. An HEV is safe during normal operations. However, it can catch a fire due to multiple conditions, namely, high temperature of the engine, a broken battery, leaking fluids, malfunctioning fuel tank, high temperature of exhaust manifolds, and abnormal wear of the engine and battery. Accidents increase the chances of fires. Therefore, it is important to understand the conditions that lead to potential fires inside the engine and battery compartments and take actions for eliminating the impacts. The research described in this paper focuses on this topic.

According to a recent publication of National Fire Protection Association (NFPA) [1], there have been nearly 287,000 vehicle fires between 2003 and 2007 in USA. The fires have claimed numerous lives and caused property damage. Most of the vehicle fires were due to automotive fluid leaks, worn-out mechanical components, collisions, and electrical failures. Engine compartment fires were about $86 \%$ of the reported minor cases and 70\% of the major cases. Engine fires were mainly due to a fuel tank or fuel line malfunctions. The high-voltage lithium-ion battery fire in a Chevy Volt passenger HEV [2] is one of the recent examples of battery fire incidents.

If the temperature and moisture in the engine compartment are high, there is a possibility of a fire. A battery is one of the main sources of energy in an HEV [3]. If the battery is not operating efficiently during the charging process, the voltage does not increase. This indicates a chemical imbalance in the battery. If this pattern continues and the temperature of the compartment increases, the battery could catch fire. Fuel leaks and mismanaged energy management could lead to engine fires in an HEV [4]. Excessive charging could influence the explosion of the battery and a potential fire. Therefore, it is important to detect and eliminate potential fires automatically.

During a typical battery charge, voltage of the battery increases at constant current input, and it decreases after it reaches the peak voltage point. Typical wet-cell lead acid 
batteries have an operating temperature between $85^{\circ} \mathrm{F}$ and $95^{\circ} \mathrm{F}$. They seem to degrade in performance if the temperature is greater than $125^{\circ} \mathrm{F}$. At this temperature, there is the possibility of chemical imbalance and a potential fire hazard.

Based on the earlier discussions, the following data identifies potential fires: the temperature and moisture percentage of the engine compartment, temperature of the battery compartment, voltage characteristics of the battery during the charging process, and the characteristics of the current charge and discharge of the battery. Sensors read data in the engine and battery compartments.

The sensor readings over a period of time show different patterns depending on the conditions of the engine and battery compartments. The synthesis of the data is required based on the time and pattern of the data. For example, in the engine compartment, the rate of change of temperature may increase, decrease, or stay at the same level for a particular length of time. In this case, the intelligent system must monitor the temperature readings over a time period from the sensor and analyze the patterns to determine if a potential fire condition exists. The rate of change can take values that are greater than zero. In general, subject matter experts express the data values in subjective terms, namely, low, medium, and high values. There is no precise definition for the values of low or high. Therefore, the traditional analytical techniques lack approaches to handle subjective linguistic terms. An intelligent system and a new approach are necessary to collect the required data and synthesize them to detect potential fires and take actions. Moreover, the system must handle linguistic definitions of the rate of change of values.

Fuzzy logic [5] provides a reasoning mechanism for synthesizing vague and uncertain linguistic parameters. In the literature, fuzzy logic has been used to detect fires in dry bay and the engine compartment of an aircraft $[6,7]$. However, they use either rule-based heuristics or analysis of histograms and images. A network-based fire detection [8] is also in the literature, but it is mainly for home automation systems. The related work in $[9,10]$ uses the traditional Mamdani fuzzy logic [11] approach for detecting fires. Most of all the fuzzy logic applications in the literature seem to use the Mamdani approach for designing a system for fire detection. The Mamdani approach allows users to express fuzzy rules of a system using linguistic terms. Therefore, the experts tend to define the rules using natural language, and it increases the complexity of a rule base. As the rule base increases, the memory space and computations required to process them increase. In addition, they all use output membership functions for approximation, and it requires more memory and computations.

A fuzzy noncontroller type of system processes fuzzy inputs and produces a deterministic output. The output is nonfuzzy, and it can have multiple deterministic values based on the rules implication. This type of system is required for detecting and eliminating potential fires in an HEV. This paper proposes a novel Fuzzy Deterministic Noncontroller Type (FDNCT) inference system and an algorithm. The FDNCT must be simple with less memory and computation requirements. It must minimize the complexity of future rule modifications. In addition, it must aid in implementing a FDNCT chip using simple architecture and minimal number of logic elements.

The work described in $[12,13]$ proposes rule reduction approaches to achieve computational efficiency. On the other hand, the authors seem to introduce complex algorithms for reducing the rules and creating a new set of membership functions from them. The approaches described in $[12,13]$ complicate the subject matter experts to define new rules or modify the existing rules. Conversely, singleton fuzzy set approaches in $[14,15]$ provide a model for using real numbers in the consequent part of a fuzzy rule and let the fuzzy inference approximate the output based on the combined weighted average of all the rule antecedents. However, they require defining multiple fuzzy singletons or real numbers to obtain the results. The weighted averages may not be the output the system is expecting to perform some actions. Therefore, additional processing or memory is required before using the output results. The approaches defined in $[16,17]$ also follow the similar approximation approaches and require defining multiple real numbers or fuzzy singletons. None of these approaches have approximation methods for producing a deterministic output using one real number or a fuzzy singleton, for example, outputting a deterministic value of 0.25 or 0.5 depending on the implication of the appropriate rule antecedents.

No simple approach exists in the literature, which is similar to the proposed FDNCT for detecting a potential fire and determining the actions for eliminating it. To fill that void and the shortcomings of the approaches described in [1417], this paper provides the following novel contributions for detecting and eliminating potential fires in the engine and battery compartments of an HEV.

(i) FDNCT system and FDNCT inference algorithm (FIA): the FDNCT and FIA processes fuzzy inputs and produces a deterministic nonfuzzy output value.

(ii) Intelligent Detection System of Potential Fires (IDSPF): architecture of the IDSPF based on the proposed FDNCT system and FIA. The FIA produces the deterministic values of initial, standby, spray, and notify actions for the IDSPF.

The IDSPF has the following distinct features that differentiate it from the existing literature described in [14-17].

(i) Subject matter experts always express rules using the Mamdani approach, and the FDNCT system approach organizes them for inference. Therefore, there is no change for the experts for adding or modifying rules.

(ii) Linguistic variables represent the rules output, namely, initial (In), standby (St), and spray (Sp), but, during the output approximation, the FIA produces one real number depending on the implication of rule antecedents. Sections 2 and 3 have the details.

(iii) The FIA is an extension and alternative for the existing fuzzy singleton inference algorithms described in [14-17] methods. Sections 2 and 3 have the details. 
(iv) The FDNCT and FIA provide simple architecture than the existing fuzzy singleton inference algorithms described in [14-17] methods. The FIA aids in developing a FDNCT chip using the minimum number of components (authors' future work).

The IDSPF continuously monitors the engine and battery compartments for the incidents of high-temperature, leaked fluids (moisture) and abnormal voltages during charging of the battery. It then synthesizes the data using FDNCT and determines the actions required, namely, spray the fire-extinguishing agent, keep the sprayer in standby or initial mode, and notify passengers using an audible alarm in the passenger compartment. IDSPF executes the actions to eliminate a potential fire.

The organization of the rest of the paper is as follows. Section 2 presents the FDNCT system model and architecture of the IDSPF. Section 3 describes the FDNCT inference algorithm (FIA). Section 4 describes an example of an FDNCT implementation. Section 5 presents the simulation results of the IDSPF using FDNCT with respect to the singleton type of approaches of detecting potential fires. This paper concludes in Section 6.

\section{Intelligent Detection System of Potential Fires (IDSPFs)}

For explaining the proposed IDSPF and FDNCT, this paper assumes rules and operating points of the engine and battery compartments of an HEV. The actual rules and the operation points of the implementation depend on the expert knowledge about the situation where IDSPF and FDNCT are applied. Each implementation can have different operating ranges, but all follow the same approach proposed in IDSPF and FDNCT.

This section describes the system architecture of the proposed IDSPF in Section 2.1 and FDNCT system model in Section 2.2.

2.1. IDSPF Architecture. Figure 1 shows the schematic of the proposed IDSPF system architecture. Components of the IDSPF are as follows:

(i) sensors (temperature (two sensors), moisture, voltage, and current),

(ii) spray jets (four),

(iii) wireless router,

(iv) intelligent fuzzy processing unit (IFPU):
(a) FDNCT system,
(b) data processor,
(c) data storage,
(d) notification/extinguishing processor,

(v) electronic alarm.

Figure 2 illustrates the process flow of the IDSPF.
(1) The following five sensors monitor the engine and battery compartments every minute and send data to the IFPU.

(i) engine compartment:
(a) temperature sensor,
(b) moisture sensor,

(ii) battery compartment:

(a) temperature sensor,

(b) voltage sensor on the battery,

(c) current sensor on the battery.

For faster temperature and moisture data acquisitions, a near infrared type of detectors [18] can be used. However, this paper does not suggest a type of sensors to use as long as the sensors meet the following IDSPF assumptions:

(i) the IDSPF uses predetermined sensors and spray jet types,

(ii) the moisture sensor sends data in percentages (\%),

(iii) the placement and location of the sensors handle various factors, namely, noise cancellation, ability to withstand high-temperature environments, sustain vibration and shock, and sense accurate data,

(iv) spray jets use predetermined fire-extinguishing agents. It meets the Underwriters Laboratories (UL) classifiers (http://www.ul.com/), namely, A for cloth, $\mathrm{B}$ for flammable liquids and gases, $\mathrm{C}$ for live electrical equipment, and $\mathrm{D}$ for combustible metals,

(v) sensors processes the signal data and converts to numerical data,

(vi) sensors transmit data using wireless communications.

(2) The sensors/detectors communicate with the IFPU using a Wireless Local Area Network (WLAN. The IFPU receives data from the sensors at the Data Processor and stores them in the Data Storage (e.g., an external hard drive or physical memory device). Let $T_{e c}$ be the current temperature reading from the engine, $M_{e c}$ be the current moisture reading from the engine, $T_{b c}$ be the current temperature reading from the battery compartment, $V_{b c}$ be the current voltage reading of the battery, and $I_{b c}$ be the present reading of the electric current of the battery.

(3) The data processor calculates the rate of change of each sensor data and stores them in the data storage every two minutes. Let $R T_{e}$ be the rate of change of temperature readings of the engine, $R M_{e}$ be the rate of change of moisture reading from the engine, $R T_{b}$ be the rate of change of temperature reading from the battery compartment, $R V_{b}$ be the rate of change of voltage readings of the battery, and $R I_{b}$ be the rate of change of readings of the electric current of the battery. 


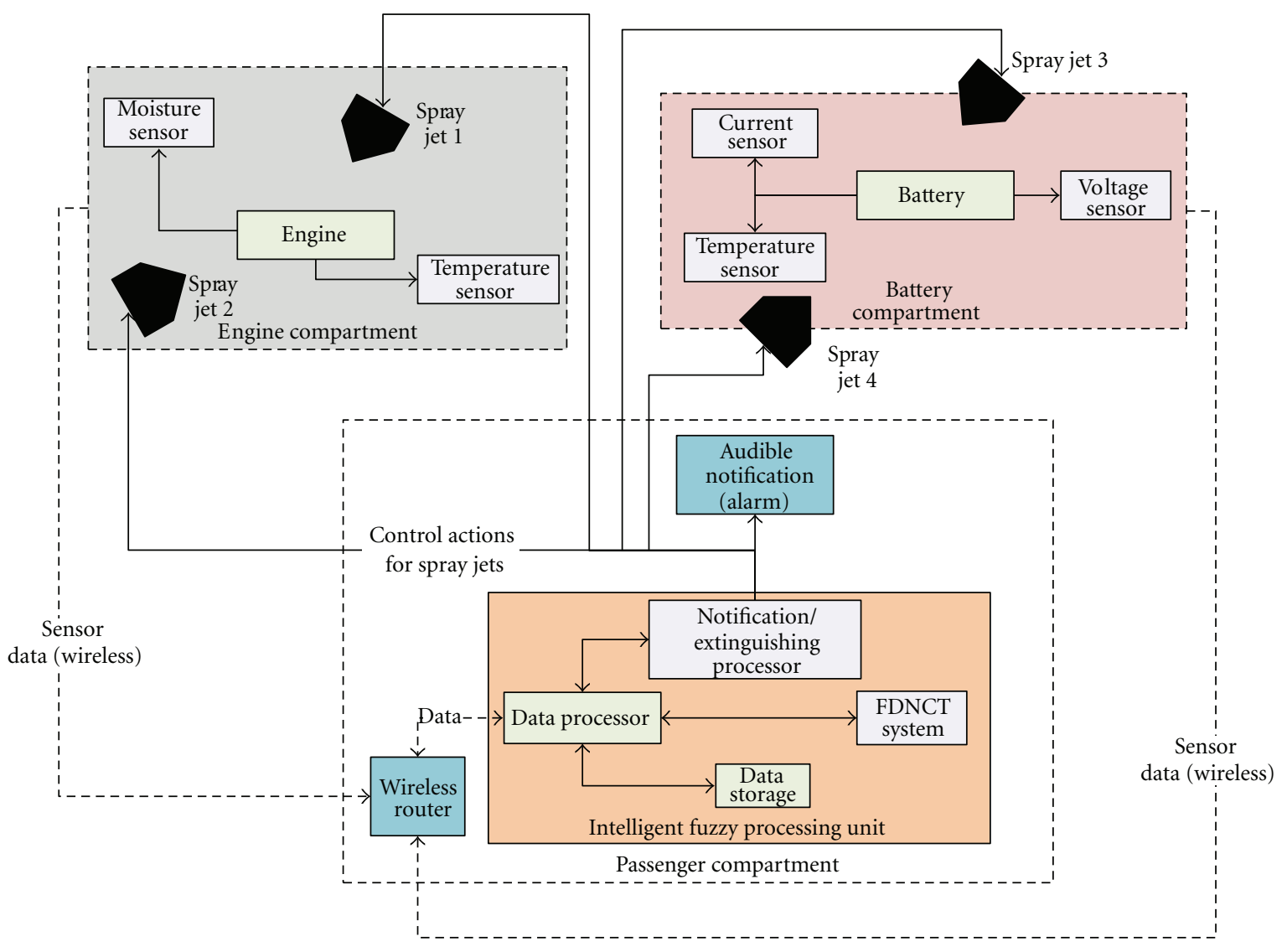

FIGURE 1: A schematic of the IDSPF system architecture.

(4) The FDNCT system receives data from the data processor and applies FIA. It sends an output back to the data processor. The FDNCT system implements the proposed FIA.

(5) Based on the readings of the sensors, the data processor interacts with the FDNCT system as follows.

(i) Send $T_{e c}$ and $M_{e c}$ to the FDNCT system and receive an output decision $O_{c}$ for the engine compartment.

(ii) Send $T_{b c}, V_{b c}$, and $I_{b c}$ to the FDNCT system and receive an output decision $O_{c 1}$ for the battery compartment.

(iii) Send $R T_{e}$ and $R M_{e}$ to the FDNCT system and receive an output decision $O_{r c}$ for the engine compartment.

(iv) Send $R T_{b}, R V_{b}$, and $R I_{b}$ to the FDNCT system and receive an output decision $O_{r c 1}$ for the battery compartment.

The data processor normalizes the sensor readings data before sending it to FDNCT system using the appropriate numbers shown in Table 2.

(6) The data processor determines the appropriate actions based on the values of $O_{c}, O_{o c 1}, O_{r c}$, and $O_{r c 1}$. The actions are to keep the spray jets in their default state, spray the fire extinguishing agent, keep the spray jets in a standby mode, and alarm the passengers about a potential vehicle fire. The deterministic output from the FDNCT system enhances the decision process of the IDSPF to take actions quickly.
(7) The notification/extinguishing processor executes the actions recommended by the data processor.

2.2. FDNCT System Model. The FDNCT is an extension and alternative for the existing fuzzy singleton inference algorithm described in [14-17]. The FDNCT system has a modified fuzzifier. The fuzzy inference of the FDNCT replaces the singleton inference algorithm. This section describes the proposed FDNCT system model used in the IDSPF for the engine (1) and battery (2) compartments of an HEV. The model is as follows:

$$
R_{e n}^{i}: \text { if } T_{e} \text { is } L^{i} \text { and } M \text { is } M^{i} \text { then } O \text { is } k^{i},
$$

where $R_{e n}^{i}$ is the $i$ th rule of $m$ rules, that is, $(1<i<m)$ of a potential fire in the engine compartment. $T_{e}$ and $M$ are the normalized engine temperature and moisture sensor data instance of the engine compartment, respectively. $L^{i}$ and $M^{i}$ are the associated linguistic input membership functions or the fuzzy sets for the temperature and moisture data, respectively, where $i=1$ to 3 , respectively. $O$ is the output. $k^{i}$ is the deterministic values $i=1$ to $3, k^{1}=1$ (initial $(\operatorname{In})$ ), $k^{2}=2$ (standby $\left.(\mathrm{St})\right), k^{3}=3$ (spray $\left.(\mathrm{Sp})\right)$ if $k^{i} \geq 2$ (notify $(\mathrm{Nt}))$ :

$R_{b a}^{i}:$ if $T_{b}$ is $A^{i}$ and $I$ is $B^{i}$, and $V$ is $C^{i}$, then $O$ is $k^{i}$, 


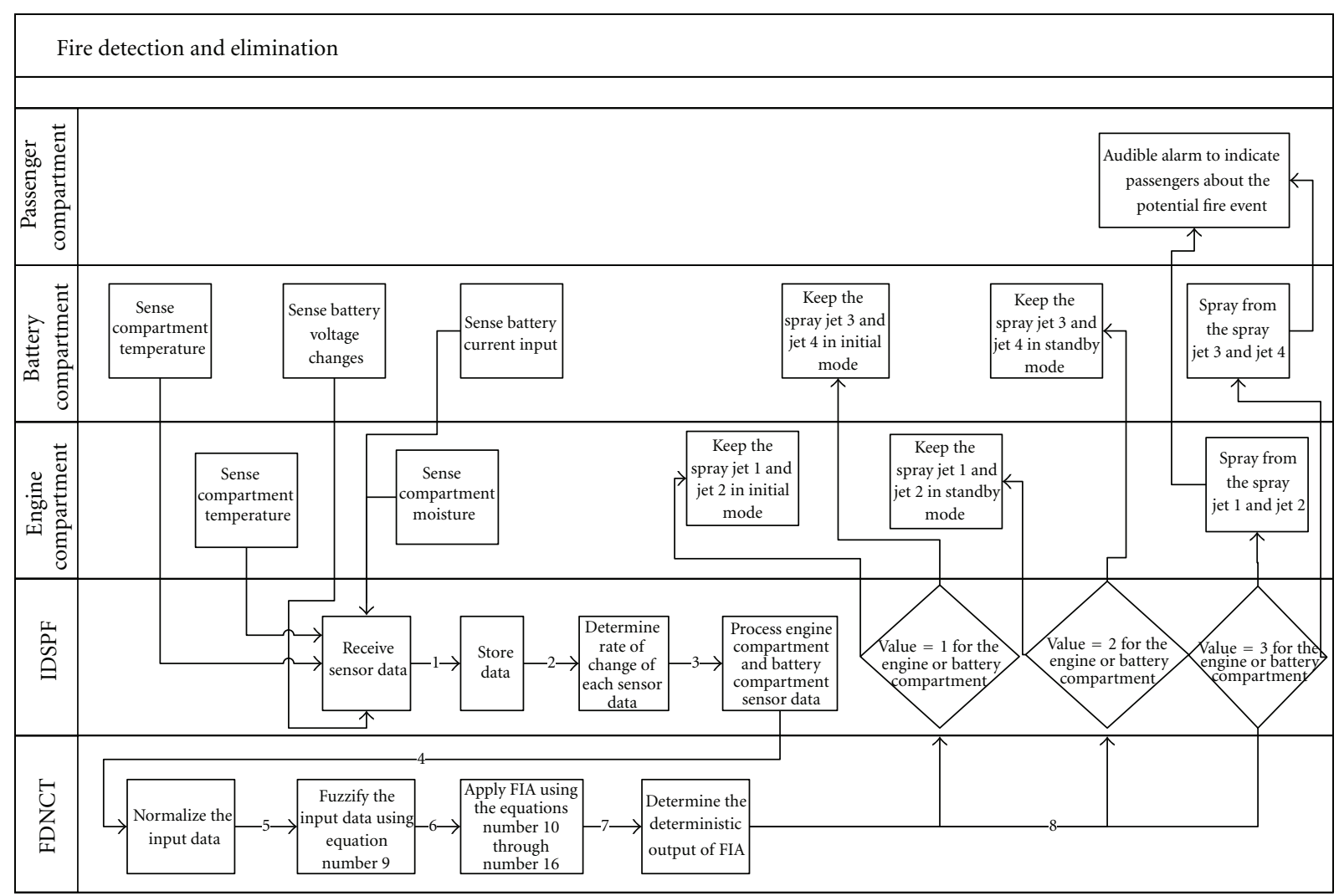

FIGURE 2: An outline of the IDSPF operation process.

TABle 1: Unique number per unique output linguistic variable.

\begin{tabular}{lc}
\hline Variable & Assigned number \\
\hline In & 1 \\
St & 2 \\
Sp & 3 \\
\hline
\end{tabular}

TABLE 2: Normalization maximum numbers.

\begin{tabular}{lccc}
\hline Variable & Maximum number & Variable & Maximum number \\
\hline$T_{e}$ & 225 & $R T_{e}$ & 100 \\
$M$ & 100 & $R M$ & 100 \\
$T_{b}$ & 125 & $R T_{b}$ & 100 \\
$V$ & 13 & $R V$ & 13 \\
$I$ & 5 & $R I$ & 5 \\
\hline
\end{tabular}

where $R_{b a}^{i}$ is the $i$ th rule of $m$ rules, that is, $(1<i<m)$ of a potential fire inside the battery compartment. $T_{b}, I$, and $V$ are the normalized engine temperature, current, and voltage sensor data instance of the battery compartment, respectively. $A^{i}, B^{i}$, and $C^{i}$ are the associated linguistic input membership functions or the fuzzy sets where $i=1$ to 3 , respectively. $O$ is the output. $k^{i}$ is deterministic values $i=1$ to $3, k^{1}=1$ (initial $\left.(\operatorname{In})\right), k^{2}=2(\operatorname{standby}(\mathrm{St})), k^{3}=3$ (spray $(\mathrm{Sp}))$ if $k^{i} \geq 2($ notify $(\mathrm{Nt}))$.
The IDSPF uses both the rate of change of input values (normalized) and the actual values (normalized) to determine the correct actions for a potential fire event

$$
\begin{aligned}
& L^{i}=M^{i}=A^{i}=B^{i}=\{\text { low, medium, high }\}, \\
& C^{i}=\{\text { no change, decrease, increase }\} .
\end{aligned}
$$

To simplify computation and to reduce processing, the linguistic input membership functions use a triangular characteristic curve. Figure 3 illustrates the triangular membership characteristic curve. The membership functions of temperature, current, and moisture inputs use the simple linguistic terms, namely, low, medium, and high. However, the names for the voltage variable are decrease, increase, and no change. In Figure $3, s, c$, and $e$ on the $x$-axis are the start, center, and end range of a fuzzy set, respectively. $\mu_{F}(a)$ on the $y$-axis is the membership grade, and $a$ is the input value to be fuzzified. The membership grade of $a$ is zero at $s$ and $e$, but at $c$ the membership grade of $a$ is 1 . The values between $s$ and $e$ have different grades of membership based on the position of $a$ and the triangle.

Unlike the approaches in the literature, the FDNCT uses no output membership functions or singletons, but the input membership functions are represented in (3) as fuzzy sets. Table 4 illustrates the normalized ranges used for the membership functions. Figure 4 illustrates the membership curves for the inputs, namely, $T_{e}, T_{b}$, and $I$. Figure 5 illustrates the membership curve for the input, namely, $V$. 
TABLE 3: FDNCT example values.

\begin{tabular}{lccccccc}
\hline Variable & Normalized & $\mu_{1}$ & $\mu_{2}$ & $\mu_{3}$ & $\max \mu$ & Index & Coefficient \\
\hline$T_{e}$ & 0.71 & 0 & 0.26 & 0.28 & 0.28 & 2 & 3 \\
$M$ & 0.75 & 0 & 0.15 & 0.38 & 0.38 & 2 & 2 \\
$T_{b}$ & 0.68 & 0 & 0.35 & 0.2 & 0.35 & 2 & 2 \\
$V$ & 0.6 & 0 & 0.58 & 0 & 0.58 & 0.43 & 3 \\
$I$ & 0.77 & 0 & 0.09 & 0.43 & & & 2.5 \\
\hline
\end{tabular}

TABLE 4: Range values of input membership functions.

\begin{tabular}{lccc}
\hline Input fuzzy set/membership & \multicolumn{3}{c}{ Normalized range values } \\
function name & $s$ & $c$ & $e$ \\
\hline Low, no change & -0.4 & 0 & 0.4 \\
Medium, decrease & 0.1 & 0.5 & 0.8 \\
High, increase & 0.6 & 1 & 1.4 \\
\hline
\end{tabular}

TABle 5: Rules developed using the Mamdani approach for the engine compartment.

\begin{tabular}{lccc}
\hline Te (temperature) & \multicolumn{3}{c}{$M$ (moisture) } \\
Low & Low & Medium & High \\
Medium & $\ln$ & $\ln$ & St \\
High & $\ln$ & St & Sp \\
\hline
\end{tabular}

Before fuzzifying the inputs, the input values are normalized based on the maximum numbers for a given variable as shown in Table 2. The fuzzifier of the FDNCT maps the nonfuzzy inputs of $T_{e}, T_{b}, M, I$, and $V$ into their suitable membership grades based on (3), Table 4, Figures 4 and 5 . This process is known as fuzzification. The process uses (4) for calculating the membership grades:

$$
\begin{aligned}
\mu_{F}(a) & =\{0 \mid a \leq s \text { or } a \geq e\} \\
& =\{1 \mid a=c\} \\
& =\left\{\frac{a-s}{c-s} \mid s \leq a \leq c\right\} \\
& =\left\{\frac{s-a}{c-s} \mid c \leq a \leq e\right\} .
\end{aligned}
$$

Fuzzy inference process of the FDNCT depends on the if-then rules defined in (1) and (2). Subject matter experts express the rules of potential fire detection and the action for eliminating them using Mamdani approach. For simplifying the rules, linguistic names are provided for each of the expected output, namely, In for the initial status of the sprayer, St for keeping the sprayer in standby mode, $S p$ for spraying the fire extinguishing agent from the sprayer, and Nt for sending alarm notification for the passengers. Tables 5 and 6 represent the Mamdani rule sets for the engine and battery compartments, respectively. The Mamdani approach requires defining membership functions for each output. The singleton model approaches in the literature use real

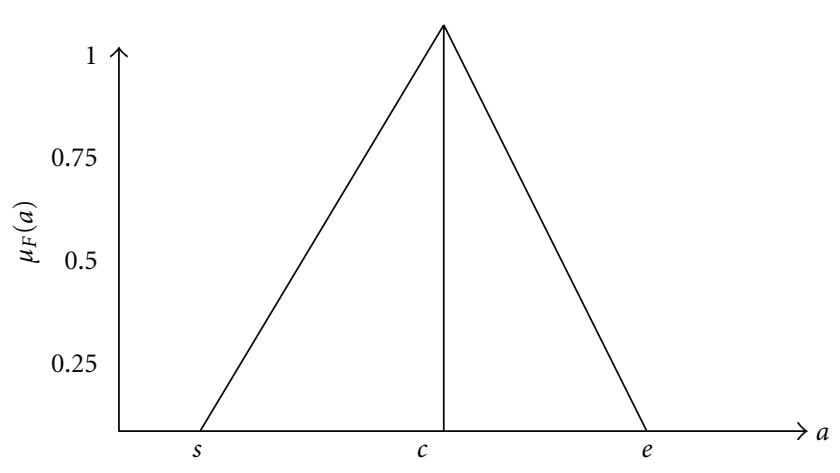

FIgURe 3: A triangular membership characterization curve.

numbers as the outputs, but they are not deterministic outputs. In contrast with the existing singleton model in the literature, the proposed FDNCT system uses no output membership function, instead it calculates the deterministic output value $k^{i}$ based on the implication of the rules in a novel way using FDNCT inference algorithm (FIA). Section 3 describes the FIA for the engine and battery compartments to determine the deterministic output value $k^{i}$.

\section{FDNCT Inference Algorithm (FIA)}

This section describes the proposed FIA. The model is expressed as shown in (5). Let $k$ be the expected output of the FDNCT system, $J$ be the output matrix based on the $m$ rules of the FDNCT system, $x_{i}$ be the $i$ th row number of the output of the $J$ matrix, and $y_{i j}$ be the $j$ th column number of the $i$ th row of the output of the $J$ matrix, where $i=1$ to $n$ rows and $j=1$ to $m$ columns of the output matrix $J$. The FIA assumes that rule implication aggregation uses fuzzy OR operator:

$$
k=J\left(x_{i}, y_{i j}\right) .
$$

The value of $x_{i}$ can be calculated using (6). Let $I_{1}$ be the value of the first input variable of the FDNCT system and $\mu_{p 1}$ and $a_{p 1}$ be the corresponding membership grade and output coefficient of the $p$ th linguistic input membership function (fuzzy set), respectively, where $p=1,2, \ldots, n$ linguistic input membership functions of the first input variable. The value of $\mu_{p 1}$ can be calculated using (4).

Let $V$ be the vector of membership grades $(\mu)$ of membership functions of the 1st input variable and $V_{a}$ be the vector of output coefficients of the corresponding 
TABLE 6: Rules developed using the Mamdani approach for the battery compartment.

\begin{tabular}{lccccccccc}
\hline \multirow{2}{*}{$T_{b}$ (temperature) } & $\begin{array}{c}\text { Low, } \\
\text { increase }\end{array}$ & $\begin{array}{c}\text { Low, } \\
\text { decrease }\end{array}$ & $\begin{array}{c}\text { Low, } \\
\text { no change }\end{array}$ & $\begin{array}{c}\text { Medium, } \\
\text { increase }\end{array}$ & $\begin{array}{c}\text { Medium, } \\
\text { decrease }\end{array}$ & $\begin{array}{c}\text { Medium, } \\
\text { no change }\end{array}$ & $\begin{array}{c}\text { High, } \\
\text { increase }\end{array}$ & $\begin{array}{c}\text { High, } \\
\text { decrease }\end{array}$ & $\begin{array}{c}\text { High, } \\
\text { no change }\end{array}$ \\
\hline Low & $\ln$ & $\ln$ & $\mathrm{St}$ & $\ln$ & $\ln$ & $\mathrm{St}$ & $\ln$ & $\ln$ & $\mathrm{St}$ \\
Medium & $\mathrm{ln}$ & $\mathrm{St}$ & $\mathrm{Sp}$ & $\mathrm{ln}$ & $\mathrm{St}$ & $\mathrm{Sp}$ & $\mathrm{ln}$ & $\mathrm{St}$ & $\mathrm{Sp}$ \\
High & $\mathrm{St}$ & $\mathrm{St}$ & $\mathrm{Sp}$ & $\mathrm{St}$ & $\mathrm{St}$ & $\mathrm{Sp}$ & $\mathrm{St}$ & $\mathrm{St}$ & $\mathrm{Sp}$ \\
\hline
\end{tabular}

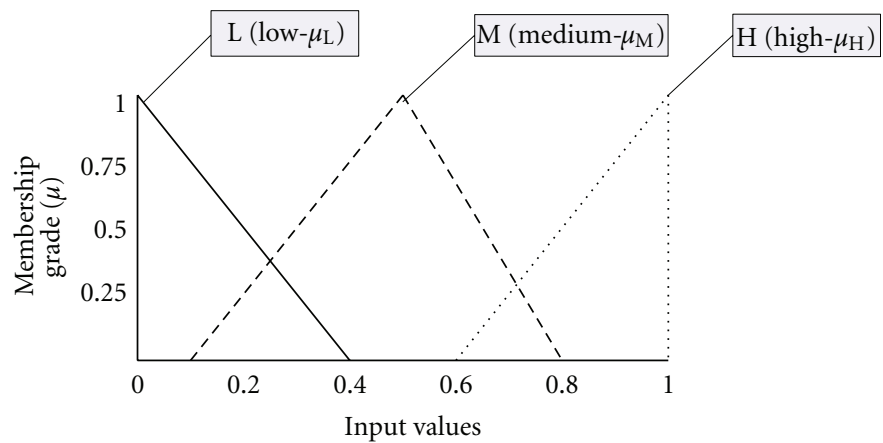

FIgURE 4: Input membership characteristic curves for the inputs $T_{e}, T_{b}$, and $I$.

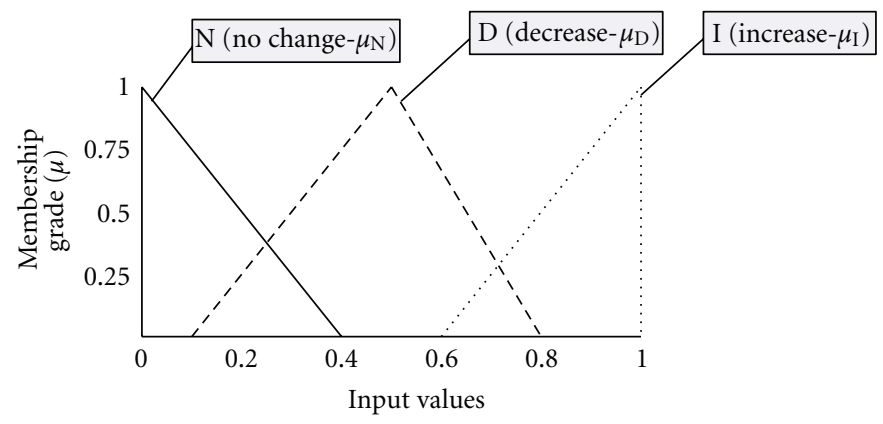

FIGURE 5: Input membership characteristic curve for the input $V$.

membership functions. Let $z$ be the index of maximum $\mu$, that is, $\mu_{\max 1}$ (8) of the 1st input variable in $V$

$$
\begin{gathered}
x_{i}=V_{a}(z), \\
V(p)=\left(\mu_{p 1}\left(I_{1}\right)\right), \\
\mu_{\max 1}=\max (V(p)) .
\end{gathered}
$$

The value of $y_{i j}$ can be calculated using (9). Let $I_{l}$ be the value of $l$ th input variable of the FDNCT system where $l=2$ to $X$ inputs, $\mu_{p l}$ and $a_{p l}$ be the corresponding membership grade and output coefficient of the $p$ th linguistic input membership function (fuzzy set) of the lth input, respectively, where $p=1,2, \ldots, n$ linguistic input membership functions. The value of $\mu_{p l}$ can be calculated using (4).

Let $W_{l}$ be the vector of membership grades $(\mu)$ of membership functions of the $l$ th input. Let $W_{a l}$ be the vector of output coefficients of the corresponding membership functions of the $l$ th input. Let $z_{l}$ be the index of maximum $\mu$, that is, $\mu_{\max l}$ (11) of the $l$ th input in $W_{l}$ :

$$
\begin{gathered}
y_{i j}=\sum_{l=2}^{X} W_{a l}\left(z_{l}\right), \\
W_{l}(p)=\left(\mu_{p l}\left(I_{l}\right)\right), \\
\mu_{\max l}=\max \left(W_{l}(1), W_{l}(2), \ldots, W_{l}(p)\right) .
\end{gathered}
$$

The FIA implementation procedure is as follows.

Step 1. Arrange fuzzy if-then rules in a matrix format as shown in Tables 5 and 6 . Let $J$ be the $n \times m$ output matrix consisting of all the outputs for the unique combinations of the membership functions, where $n$ is the number of rows and $m$ is the number of columns. As shown in Table 6, the assumption is that $J$ can have only one linguistic input membership variable associated with an output per row and multiple linguistic input variables per column. The experts 
TABLE 7: Battery compartment rule matrix with numerical outputs, linguistic inputs, and output coefficients.

\begin{tabular}{|c|c|c|c|c|c|c|c|c|c|}
\hline \multirow{3}{*}{$T_{b}$ (temperature) } & \multicolumn{9}{|c|}{$I-V($ current and voltage $)$} \\
\hline & 1 & 2 & 3 & 1 & 22 & 2 & 3 & 3 & 3 \\
\hline & {$[0.5][0.5]$} & {$[0.5][1.5]$} & {$[0.5][2.5]$} & {$[3.5][0.5]$} & [3.5] [1.5] & {$[3.5][2.5]$} & {$[6.5][0.5]$} & {$[6.5][1.5]$} & [6.5] [2.5] \\
\hline $1[1]$ & 1 & 1 & 2 & 1 & 1 & 2 & 1 & 1 & 1 \\
\hline $2[2]$ & 1 & 2 & 3 & 1 & 2 & 3 & 1 & 2 & 3 \\
\hline $3[3]$ & 2 & 2 & 3 & 2 & 2 & 3 & 2 & 2 & 3 \\
\hline
\end{tabular}

express linguistic variables as low, high, increase, and so forth.

Step 2. Identify unique linguistic outputs in $J$ and assign unique numbers starting from 1 . Let $\lambda$ be the total number of unique outputs. Tables 5 and 6 illustrate the result of this step for the outputs as shown in Table 1. Replace all the linguistic output variables in $J$ with the assigned unique numbers $1,2,3, \ldots, \lambda$. Let $\alpha_{i j}$ be the assigned output number for the $i$ th row and $j$ th column of $J$.

Step 3. For each input in $J$, assign a unique number to each unique linguistic input variable using an increment of one starting from one. Table 7 illustrates an example assignment.

Step 4. Let $\vartheta_{j}$ be the total number of linguistic input variables in the $j$ th column that have no output coefficients and $\theta_{j}$ be the total number of linguistic input variables that have output coefficients. Let $a_{i}$ be the output coefficient of a linguistic input variable, where $i$ is 1 to $\theta_{j}$. Determine delta output coefficient $\xi_{j}$ using (12) and assign it to all the linguistic input variables that have no output coefficients in the $j$ th column. If any of the remaining columns have any linguistic input variables in the same positions as the $j$ th column, then assign their output coefficients with the output coefficients of the corresponding linguistic input variables in the $j$ th column. For example, assume that the 1st column has low and high linguistic input variables and 0.33 and 1.35 are the output coefficients of low and high linguistic variables, respectively. Assume that 3rd column has low and medium, and 4th column has medium and high linguistic input variables. In this situation, the low variable in 3rd column gets 0.33 , and the high value in the 4 th column gets 1.35. Repeat Step 4 for all the remaining columns to make sure all the linguistic input variables have output coefficients. After performing all the assignments, the final matrix looks as shown in Figure 6 and Table 7:

$$
\xi_{j}=\frac{j-\sum_{i=1}^{\theta_{j}} a_{i}}{\vartheta_{j}} \mid j=1,2,3, \ldots, m \text { columns. }
$$

Step 5. Let $\vartheta_{k}$ be the total number of linguistic input variables in the $k$ th row that has no output coefficients and $\theta_{k}$ be the total number of linguistic input variables that have output coefficients. Let $a_{i}$ be the output coefficient of a linguistic input variable where $i$ is 1 to $\theta_{k}$. Determine delta output coefficient $\xi_{k}$ using (13) and assign it to all the linguistic input variables that have no output coefficients in the $k$ th row. Since a row can have only input variable, repeat Step 5

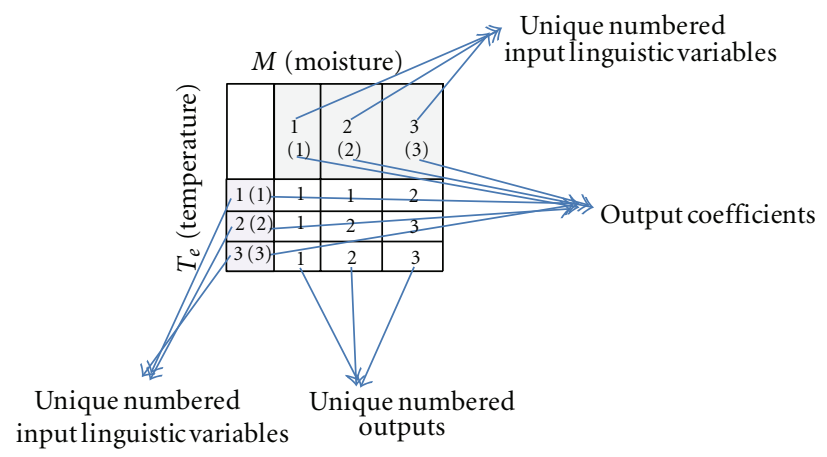

Figure 6: Engine compartment rule matrix with numerical outputs, linguistic inputs, and output coefficients.

for all the remaining rows to make sure that all the linguistic input variables have output coefficients. After performing all the assignments, the final matrix looks as shown in Figure 6 and Table 7:

$$
\xi_{k}=\frac{k-\sum_{i=1}^{\theta_{k}} a_{i}}{\vartheta_{k}} \mid k=1,2,3, \ldots, n \text { rows. }
$$

Step 6. As shown in (14), let $N$ be the total number of inputs of an FDNCT system. Let $I_{i}$ be the vector of all the numerically assigned linguistic input variables of the $i$ th input (Step 3), where $i=1,2,3, \ldots, N$. Let $\lambda_{i}$ be the total number of linguistic input variables of the $i$ th input. Let $a_{i}$ be the output coefficient of the ith linguistic input variable. Based on (14), the engine and battery compartment linguistic input variables of the IDSPF can be represented as (15) and (16), respectively. We have

$$
I_{i}(N)(N)=\left(1,2, \ldots, \lambda_{i}\right)\left(a_{1}, a_{2}, \ldots, a_{N}\right) \mid i=1,2,3, \ldots, N,
$$

$$
\begin{gathered}
I_{T_{e}}(3)=(1,2,3)(1,2,3), \\
I_{M}(3)=(1,2,3)(1,2,3), \\
I_{T_{b}}(3)=(1,2,3)(1,2,3), \\
I_{I}(3)=(1,2,3)(0.5,3.5,6.5), \\
I_{V}(3)=(1,2,3)(0.5,1.5,2.5) .
\end{gathered}
$$

The output matrix $J$ (Figure 6 and Table 7), (15), and (16) serve as the knowledge for inferring the output of an FDNCT system in the IDSPF. The following equations serve 
as the FIA engine: (4), (5), and (9). Section 4 describes the application of FIA using an example.

\section{FIA Example}

This section describes the application of FIA using an example of the inputs of IDSPF.

Example 1. We have the following steps.

Step 1. Let $T_{e}$ is $160^{\circ}$ Fahrenheit, $M$ is $75 \%, T_{b}$ is $85^{\circ}$ Fahrenheit, $I$ is 3 amperes, and $V$ is 10 volts. Normalize the inputs by dividing the value of the inputs using the appropriate number shown in Table 2 and rounding it to two decimal points. The table expresses the maximum operating point per variable used in the IDSPF implementation. The normalized values are as follows: $T_{e}=0.71, M=0.75, T_{b}=0.68, I=0.6$, $V=0.77$.

Step 2. Calculate the $\mu$ of each input value using (4) for all its corresponding input membership functions. Find the maximum value of $\mu$ value for each input, and find the associated output coefficients from (15) and (16). The calculated values are as shown in Table 3.

Step 3. Based on the values of the coefficient column in Table 3, the FDNCT output values of the engine and battery compartments can be calculated using (5). From Table 3, the inputs of the engine compartment are $T_{e}$ and $M$. The corresponding output coefficients are $x_{i}=2$ and $y_{i j}=3$; based on (5) and Figure 6 the value of $k$ is 3; that is, spray the sprayer, and since $k$ is $>2$, send the alarm notification to the passenger compartment. Similarly, for the battery compartment, $x_{i}=2$ and $y_{i j}=1.5+3.5=5$ (5th column). Based on (5) and Table 7, the value of $k$ is 2; that is, keep the sprayer in the standby mode.

\section{Simulation Results}

The authors simulated the performance of the FIA and singleton approaches using a computer software, namely, Matlab and Simulink, and a set of normalized input data for the engine and battery compartments. Table 8 illustrates a small set of the bigger set of sample data used for the simulation. The data is generated using the random() function in the Matlab library. In the future work, a fuzzy chip will be implemented to test the approach in a real set up.

Figure 7 illustrates the performance of the FDNCT over singleton approach for one sample data over 20 iterations of the engine compartment of an HEV. The FDNCT takes at an average of 0.09 seconds to perform one action for the engine compartment, whereas the singleton approach takes an average of 0.12 seconds. The FDNCT approach reduces approximately $25 \%$ of time than that of the singleton approach.

Figure 8 illustrates the performance of the FDNCT over singleton approach for one sample data over 20 iterations of the battery compartment of an HEV. The FDNCT takes an average of 0.11 seconds to perform one action for the
TABLE 8: Sample data snapshot used for the engine and battery compartments.

\begin{tabular}{llc}
\hline$T_{b} / T_{e}$ & $M, I$ & $V$ \\
\hline 0.08 & 0.14 & 0.14 \\
0.16 & 0.27 & 0.27 \\
0.18 & 0.32 & 0.32 \\
0.18 & 0.32 & 0.32 \\
0.18 & 0.32 & 0.32 \\
0.25 & 0.44 & 0.44 \\
0.31 & 0.54 & 0.54 \\
0.35 & 0.62 & 0.62 \\
0.38 & 0.67 & 0.67 \\
0.40 & 0.70 & 0.70 \\
0.40 & 0.70 & 0.70 \\
0.38 & 0.67 & 0.67 \\
0.35 & 0.61 & 0.61 \\
0.30 & 0.53 & 0.53 \\
0.25 & 0.43 & 0.43 \\
0.18 & 0.32 & 0.32 \\
0.18 & 0.32 & 0.32 \\
0.11 & 0.19 & 0.19 \\
0.03 & 0.05 & 0.05 \\
\hline$T_{i}: 19$ & &
\end{tabular}

$T_{e}$ : engine temperature, $T_{b}$ : battery temperature, $I$ : battery current, and $V$ : battery voltage.

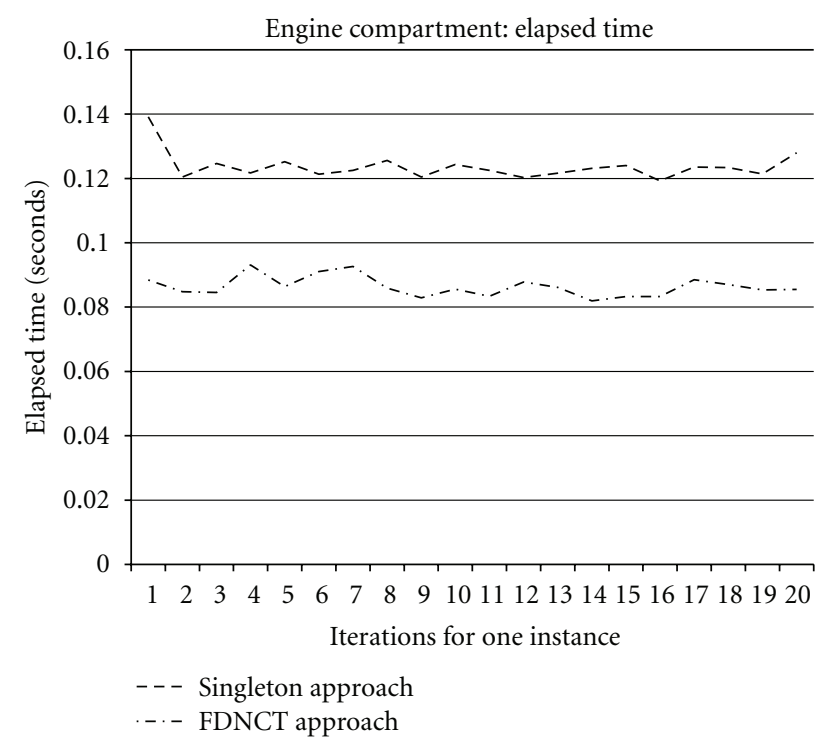

Figure 7: Elapsed time for executing one data sample for the engine compartment using the FDNCT and singleton approach.

battery compartment whereas the singleton approach takes an average of 0.19 seconds. The FDNCT approach reduces approximately $42 \%$ of time more than that of the singleton approach.

The main benefits from the FDNCT approach are to develop a fuzzy chip with minimizing the number components, and to produce deterministic outputs with a simple and minimum number of fuzzy rules. The elapsed time 


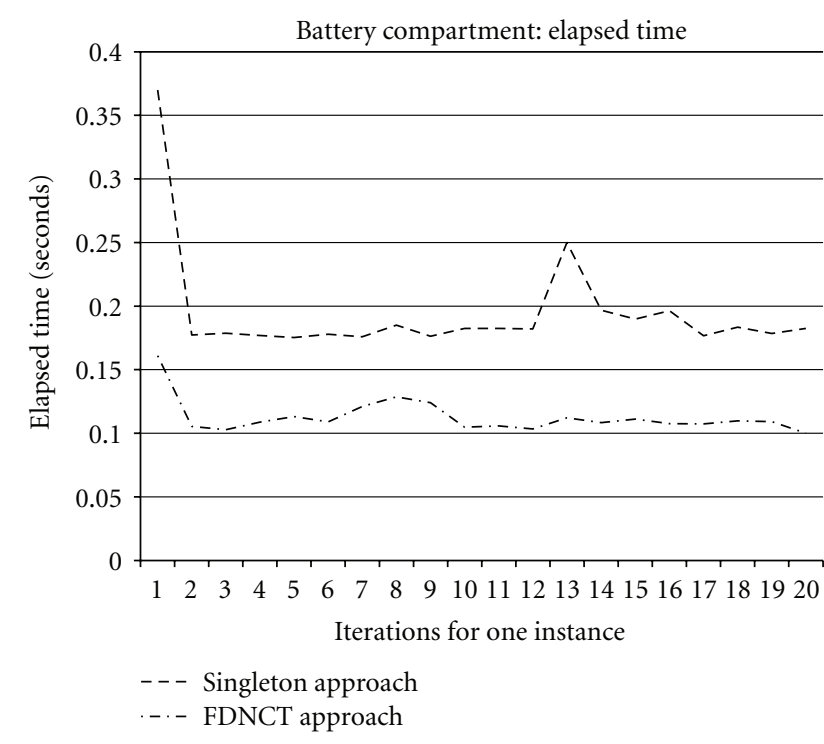

FIGURE 8: Elapsed time for executing one data sample for the battery compartment using the FDNCT and singleton approach.

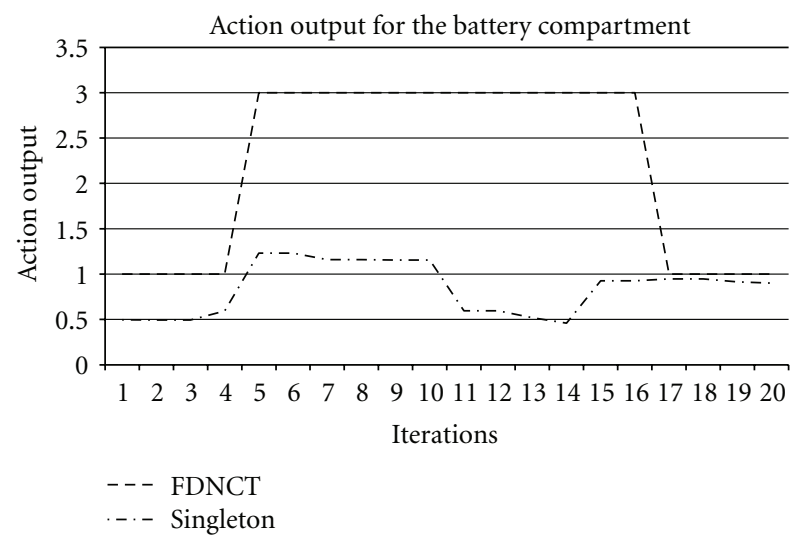

Figure 9: Action output for the battery compartment using the FDNCT and singleton approach.

advantage of the FDNCT over the singleton approach is secondary.

Figure 9 illustrates the action outputs using the FDNCT and singleton approach for 20-sample data of the battery compartment of an HEV. The FDNCT outputs deterministic values whereas the singleton approach outputs nondeterministic values. The FDNCT has crisp outputs when compared to singleton approach. The deterministic outputs are necessary for the noncontroller type of applications. Similar results were obtained for the engine compartment simulation too. Based on Figure 9, it is seen that the FDNCT approach takes spraying actions (i.e., action output $=3$ ) for iterations numbers 6 through 17. However, the singleton approach takes a very different action for each of the iterations, and it never takes the spraying action throughout the simulation. The singleton approach seems not to detect a potential fire even if the potential fire situation exists.

\section{Conclusion}

The IDSPF is a noncontroller type of system that uses fuzzy inputs and produces a deterministic output. The IDSPF uses the consequent parts of the fuzzy rules as a detection of a potential fire and the deterministic output as an action for eliminating the potential fire. For noncontroller type of systems, when compared with the fuzzy singleton approach, the FDNCT and FIA provide simple solutions with a reduced number of computations. The FDNCT produces a deterministic output without using an output fuzzy set or a membership function. The FIA and FDNCT work well with most of the noncontroller type applications that use fuzzy inputs and require a deterministic output. The FIA is an extension and alternative to the fuzzy singleton algorithm. The weighted average approach of the traditional Mamdani singleton method requires more processing and is more time consuming as the number of fuzzy rules increases. Therefore, the proposed FDNCT and FIA provide a system and an algorithm that requires less storage space and are more efficient to synthesize fuzzy inputs and to produce a deterministic output.

The authors of this paper intend to implement IDSPF and develop an FDNCT fuzzy chip using a hardware description language (HDL) in their future work. The new approach allows using minimal.

\section{Disclaimer}

Reference herein to any specific commercial company, product, process, or service by trade name, trademark, manufacturer, or otherwise does not necessarily constitute or imply its endorsement, recommendation, or favoring by the United States Government or the Department of the Army (DoA). The opinions of the authors expressed herein do not necessarily state or reflect those of the United States Government or the DoA and shall not be used for advertising or product endorsement purposes.

\section{References}

[1] M. Ahrens, "U.S. Vehicle fire trends and patterns," NSFA Report, 2010.

[2] NHTSA, "2011 chevrolet volt battery fire incident report regarding NHTSA testing and analysis OF subject vehicle HV battery fires," NHTSA Investigation Report, 2012.

[3] J. Bauouth and A. Kuznetsov, "Automotive and fire safety research," Fire Safety Library and Data Analysis, pp. 1-97, 2005.

[4] J. Moreno, M. E. Ortúzar, and J. W. Dixon, "Energy-management system for a hybrid electric vehicle, using ultracapacitors and neural networks," IEEE Transactions on Industrial Electronics, vol. 53, no. 2, pp. 614-623, 2006.

[5] L. A. Zadeh, "Is there a need for fuzzy logic?" Information Sciences, vol. 178, no. 13, pp. 2751-2779, 2008.

[6] S. Y. Foo, "A rule-based machine vision system for fire detection in aircraft dry bays and engine compartments," Knowledge-Based Systems, vol. 9, no. 8, pp. 531-540, 1996. 
[7] S. Y. Foo, "A fuzzy logic approach to fire detection in aircraft dry bays and engine compartments," IEEE Transactions on Industrial Electronics, vol. 47, no. 5, pp. 1161-1171, 2000.

[8] K. C. Lee and H. H. Lee, "Network-based fire-detection system via controller area network for smart home automation," IEEE Transactions on Consumer Electronics, vol. 50, no. 4, pp. 10931100, 2004.

[9] H. C. Mueller and A. Fischer, "Robust fire detection algorithm for temperature and optical smoke density using fuzzy logic," in Proceedings of the 29th Annual 1995 International Carnahan Conference on Security Technology, pp. 197-204, October 1995.

[10] J. M. Xiao and X. H. Wang, "A fuzzy neural network approach to fire detection in ships," in IEEE International conference on Fuzzy Systems, pp. 1459-1461, usa, May 2003.

[11] E. H. Mamdani, "Application of fuzzy logic to approximate reasoning using linguistic synthesis," IEEE Transactions on Computers, vol. 26, no. 12, pp. 1182-1191, 1977.

[12] C. W. Tao, "A reduction approach for fuzzy rule bases of fuzzy controllers," IEEE Transactions on Systems, Man, and Cybernetics, Part B, vol. 32, no. 5, pp. 668-675, 2002.

[13] Y. Yam, P. Baranyi, and C. T. Yang, "Reduction of fuzzy rule base via singular value decomposition," IEEE Transactions on Fuzzy Systems, vol. 7, no. 2, pp. 120-132, 1999.

[14] M. Sugeno and T. Yasukawa, "Fuzzy-logic-based approach to qualitative modeling," IEEE Transactions on Fuzzy Systems, vol. 1, no. 1, pp. 7-30, 1993.

[15] M. Sugeno, "On stability of fuzzy systems expressed by fuzzy rules with singleton consequents," IEEE Transactions on Fuzzy Systems, vol. 7, no. 2, pp. 201-224, 1999.

[16] M. Mizumoto, "Product-sum-gravity method-fuzzy singleton-type reasoning method-simplified fuzzy reasoning method," in Proceedings of the 5th IEEE International Conference on Fuzzy Systems, vol. 3, pp. 2098-2102, September 1996.

[17] H. Seki and M. Mizumoto, "On the equivalence conditions of fuzzy inference methods-Part 1: basic concept and definition," IEEE Transactions on Fuzzy Systems, vol. 19, no. 6, pp. 10971106, 2011.

[18] T. Meitzler, D. Bryk, E. Sohn et al., "An infrared solution to a national priority NASA ice detection and measurement problem," in Infrared Technology and Applications XXXIII, vol. 6542 of Proceedings of SPIE, Orlando, Fla, USA, April 2007. 

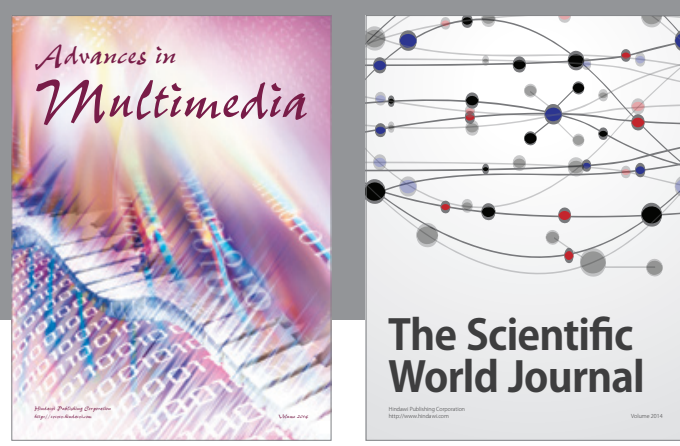

The Scientific World Journal
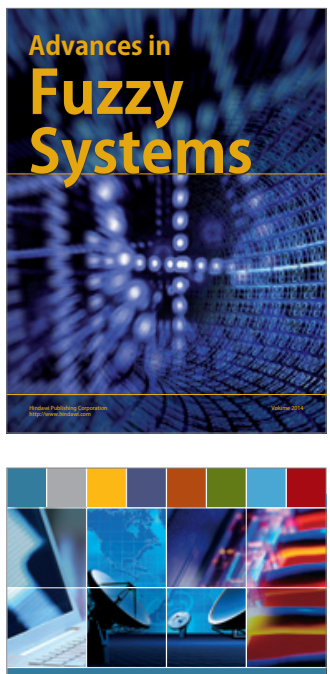

Computer Networks and Communications
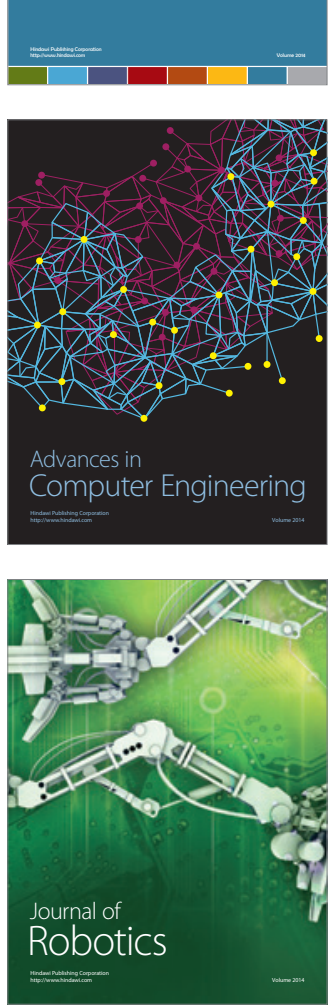
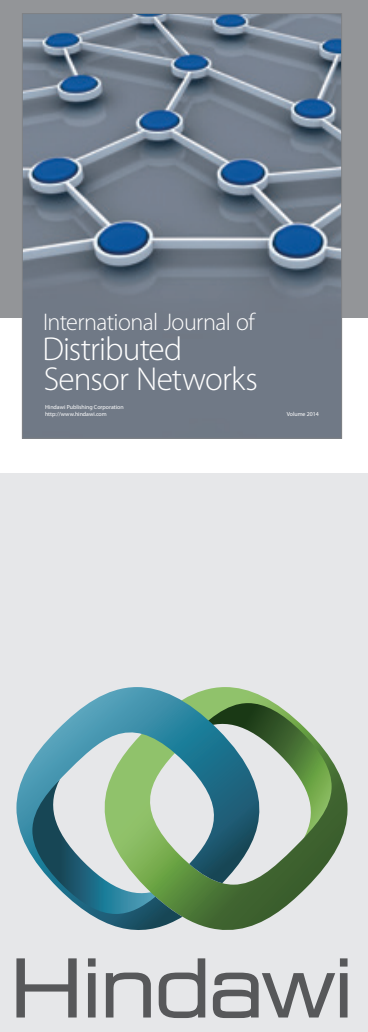

Submit your manuscripts at

http://www.hindawi.com
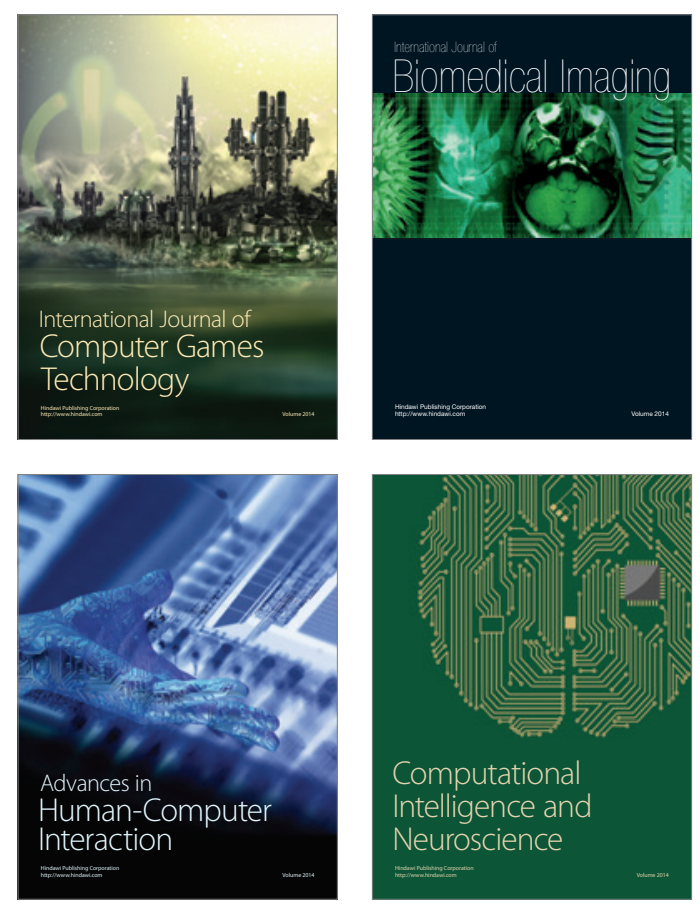
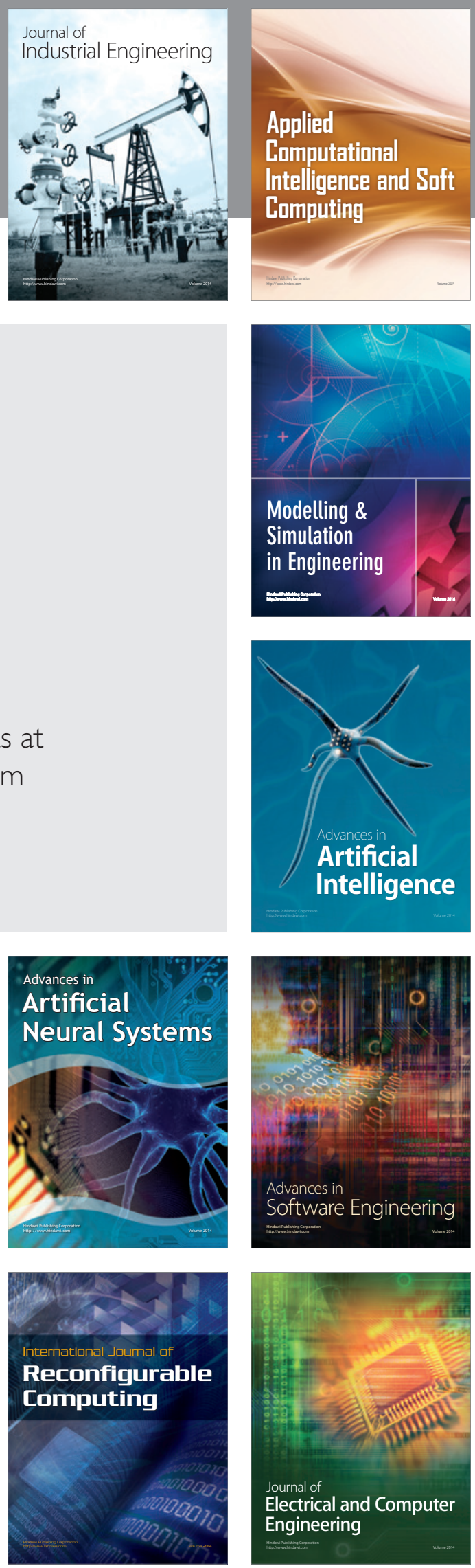